\title{
Coactivators for a proline-rich activator purified from the multisubunit human TFIID complex
}

\author{
Naoko Tanese, B. Franklin Pugh, and Robert Tjian \\ Howard Hughes Medical Institute, Department of Molecular and Cell Biology, University of California at Berkeley, \\ Berkeley, California 94720 USA
}

The mechanisms of transcriptional activation directed by sequence-specific regulators is central to understanding gene regulation. Here, we report the isolation of coactivators responsible for mediating transcriptional activation by Gal4-Pro, a hybrid regulator containing the proline-rich activation domain of human CTF/NFI. Chromatographic studies indicate that endogenous human TFIID consists of a multisubunit complex containing the TATA-binding protein (TBP), coactivators, and other associated factors. A fraction containing the coactivator activity was separated from the endogenous TBP after disrupting the tightly associated complex with urea. The urea-purified TBP was active for basal level transcription but no longer could support activation by Gal4-Pro. However, when the two separated components were added together, activated levels of transcription were restored in the presence of Gal4-Pro. Immunoaffinity purification of the TFIID complex identifies several polypeptides specifically associated with the endogenous TBP, some or all of which function as coactivators when reconstituted with Gal4-Pro. The isolated coactivators also mediate activation by a chimeric glutamine-rich activator derived from Sp1 but not the Gal4-VP16 activator, suggesting distinct factor requirements for different types of transcriptional regulators.

[Key Words: TFIID complex; proline-rich activator; transcriptional coactivators]

Received September 19, 1991; revised version accepted October 16, 1991.

Transcriptional initiation by RNA polymerase II at eukaryotic promoters is thought to involve the action of two distinct classes of transcription factors. One group comprises the general factors that are required for directing accurate initiation of transcription at all RNA polymerase II promoters (Sawadogo and Sentenac 1990), whereas the other class consists of promoter-selective regulators that modulate the activity of the general factors at specific promoters (Mitchell and Tjian 1989). The promoter-selective class includes sequence-specific transcriptional activators that bind to DNA elements located upstream or downstream of the initiation site and influence the rate of transcription. Interestingly, many sitespecific regulators studied thus far contain activation domains characterized by a high content of acidic, glutamine, or proline residues (Mitchell and Tiian 1989). Although a great deal of effort has been devoted to identifying the target of these site-specific regulators (Berger et al. 1990; Kelleher et al. 1990; Stringer et al. 1990; Lin and Green 1991), their mechanism of action remains poorly understood.

At least six general factors, TFIIA, TFIIB, TFIID, TFIIE, TFIIF, and TFIIH, isolated from HeLa cells are required in addition to RNA polymerase II to reconstitute accurate initiation of transcription in vitro (Reinberg and Roeder 1987; Reinberg et al. 1987; Buratowski et al. 1989; D. Reinberg, pers. comm.). The exact function of these basal factors in promoting transcription remains to be elucidated. However, TFIID is thought to play a pivotal role by recognizing the TATA box element normally positioned near the transcriptional start site and thereby recruiting other factors such as TFIIA and TFIIB to the promoter to nucleate the formation of a preinitiation complex (Van Dyke et al. 1988; Buratowski et al. 1989). Recent molecular cloning and characterization of the cDNA encoding the TATA-binding protein (TBP) from various organisms (Cavallini et al. 1989; Hahn et al. 1989; Horikoshi et al. 1989a; Schmidt et al. 1989; Hoey et al. 1990; Hoffman et al. 1990a; Kao et al. 1990; Peterson et al. 1990) has confirmed that TBP is responsible for binding to the TATA box and interacting with the general factors TFIIA and TFIIB to direct basal level transcription. However, we and others have found that the purified recombinant TBP could not substitute for the endogenous TFIID fraction to mediate transcriptional activation directed by promoter-specific activators such as $\mathrm{Spl}$ and upstream stimulatory factor (USF) (Hoffman et al. 1990b; Peterson et al. 1990; Pugh and Tjian 1990). Thus, a novel class of transcription factors, termed coactivators, present in the TFIID fraction has been proposed to serve as intermediaries or cofactors necessary to mediate transcriptional activation by interacting with the activator and TBP. A separate study conducted with Drosophila embryo extracts reveals that endogenous 
TBP exists as a multisubunit complex with tightly associated polypeptides (termed TAFs for TBP-associated factors) that include coactivators for glutamine-rich activators NTF-1 and Sp1 (Dynlacht et al. 1991). Interestingly, although the general transcription apparatus appears to be highly conserved from yeast to man, TBP in Saccharomyces cerevisiae does not appear to be part of a tightly associated heteromeric complex (Buratowski et al. 1988; Cavallini et al. 1988; Horikoshi et al. 1989b), as it does in higher eukaryotes.

Several lines of evidence suggest that the endogenous human TFIID is present as a multisubunit complex that includes TBP and the coactivators. For example, the endogenous human TFIID activity chromatographs in the excluded volume of an S-300 gel filtration column (predicted molecular mass $>300 \mathrm{kD}$; Pugh and Tjian 1990). In contrast, the recombinant human TBP overexpressed in HeLa cells behaves on gel filtration columns as a 40 $\mathrm{kD}$ monomer (Peterson et al. 1990). It has also been reported that the binding of TFIID to some promoters gives rise to an extended DNase I footprinting pattern $(60-80$ bp) that includes the TATA box and downstream sequences (Nakajima et al. 1988), whereas the footprint pattern of the purified recombinant TBP is confined to a 20-bp stretch containing the TATA box sequences (Peterson et al. 1990). Furthermore, electrophoretic mobility-shift assays have shown that TBP forms one specific protein-DNA complex, whereas the endogenous TFIID fraction gives rise to multiple protein-DNA complexes migrating with slower electrophoretic mobility (N. Tanese, unpubl.). Because the endogenous human TBP and the coactivator activities appear to copurify over multiple chromatographic columns (Pugh and Tjian 1990), it seemed reasonable to test the hypothesis that these two activities are part of a tightly associated multimeric TFIID complex.

To probe the mechanism of transcriptional activation mediated by TBP and coactivators in mammalian cells, we have addressed the following specific questions: Do activators other than the glutamine-rich activator $\mathrm{Sp} 1$ require coactivators for function? Are coactivators and the TBP distinct activities in mammalian cells that can be biochemically fractionated? If so, can we separate these activities physically and reconstitute activator-dependent transcription with the fractionated components? Finally, are there multiple coactivators associated with the endogenous TBP, and could they mediate the activity of different types of sequence-specific regulators? Here, we have determined that a chimeric activator, Gal4-Pro, requires TBP-associated coactivators for transcriptional activation. In addition, we have attempted to separate the endogenous TFIID complex into a coactivator component and TBP and reconstitute transcriptional activation by Gal4-Pro with the fractionated components. We have also tested the TBP-associated proteins eluted from specific immunoprecipitates of the TFIID complex for coactivator activity. Finally, we have carried out experiments with activators bearing either a glutamine-rich or acidic activation domain to determine whether different classes of activators function with different coactivators.

\section{Results \\ A proline-rich activator also requires coactivators}

To study the mechanism of action by a promoter-selective activator we constructed a chimeric protein containing the DNA-binding domain of the yeast Gal4 protein (Keegan et al. 1986) fused to the proline-rich activation domain of the human CCAAT box-binding transcription factor (CTF/NF-I; Jones et al. 1987; Santoro et al. 1988). We found that the Gal4-Pro fusion protein is a potent site-specific activator that stimulated transcription 15to 20 -fold above basal levels (Fig. 1A, lanes 2,4,6) in a binding site-dependent manner (cf. lanes 5 and 6) from a promoter containing the E4 TATA box and five Gal4binding sites. Thus, the proline-rich domain of CTF-1 is a strong transcriptional activator in vitro when attached to the DNA-binding domain of Gal4.

Previously, we reported that transcription factor $\mathrm{Spl}$ appears to require factors in the human TFIID fraction distinct from the TBP for activation in reconstituted reactions (Peterson et al. 1990; Pugh and Tjian 1990). We were therefore prompted to determine the requirements for the Gal4-Pro activator in a reconstituted transcription reaction containing RNA polymerase II and the general factors. The TFIID fraction restores a basal level of transcription that is substantially stimulated by the addition of Gal4-Pro (Fig. 1B, lanes 5,6). In contrast, when purified recombinant TBP was substituted for the TFIID fraction, basal level transcription was restored but no activation was observed with the Gal4-Pro activator (Fig. 1B, lanes 3,4). The same result was obtained with recombinant TBP made in HeLa cells by a recombinant vaccinia virus expressing TBP (data not shown). We conclude from these experiments that the Gal4-Pro activator requires the endogenous TFIID fraction for transcriptional activation in a reconstituted HeLa cell extract and that the purified recombinant TBP is unable to substitute for the TFIID fraction. To test the possibility that the recombinant TBP requires additional activities (coactivators) in the TFIID fraction to respond to the Gal4Pro activator we added the recombinant TBP to a nuclear extract whose endogenous TBP activity had been selectively destroyed by heat treatment (Fig. 1C). Under these conditions we find that both the recombinant TBP and the TFIID fraction restore basal transcription that can be stimulated by the addition of the Gal4-Pro activator (lanes 3-6). This experiment demonstrates that the recombinant TBP is capable of supporting activation by the Gal4-Pro activator if the proper coactivators are provided in the reaction. Taken together with our result from the reconstituted system, this finding strongly suggests that one or more coactivators in the TFIID fraction are required to mediate activation by the Gal4-Pro activator.

\section{Chromatographic separation of coactivators from the TFIID complex}

We have found that during conventional chromatographic fractionation of human TFIID, the coactivator 
Figure 1. Transcriptional activation by a chimeric Gal4-Pro activator requires coactivators. (A) Gal4-Pro is a potent site-specific activator in HeLa nuclear extract. The chimeric Gal4-Pro protein is a fusion betwen the amino-terminal 94 amino acids of Gal4, which is sufficient for dimerization and specific binding to DNA, and the carboxy-terminal 100 amino acids of CTF- 1 , which contain the activation region characterized by an unusually high content $(\sim 25 \%)$ of proline residues (Mermod et al. 1989). Runoff transcription reactions were performed in a crude nuclear extract in the presence of no exogenously added activator (lanes 1,2 ), control Gal4 protein containing the first 94 amino acids of Gal4 (lanes 3,4) or Gal4-Pro fusion protein (lanes 5,6$)$. Template DNAs with a TATA box from the adenovirus $\mathrm{E} 4$ promoter containing $\left(\mathrm{G}_{5} \mathrm{E} 4 \mathrm{~T}\right)$ or lacking (E4T) five Gal4-binding sites were used as indicated above each lane. Activators and template DNAs used in the experiment are shown schematically below. The specific runoff transcript of 260 nucleotides is indicated by an arrow. Note that the transcription level in the absence of the added activator is very low. Thus, by using the Gal4-Pro hybrid protein, we can effectively eliminate the influence of endogenous CTF/NF-I in our HeLa cell transcription fractions, which can complicate the interpretation and quantitation of activation directed by exogenously added transcriptional activators. $(B)$ Gal4-Pro mediates activation with HeLa TFIID fraction but not with recombinant hTBP in reconstituted transcription. Transcription reactions were reconstituted on a $\mathrm{G}_{5} \mathrm{BCAT}$ template containing a TATA box from adenovirus E1B promoter and five Gal4-binding sites, and the products were detected by primer extension analysis (indicated by an arrow). No TBP (lanes 1,2), $0.6 \mathrm{ng}$ of purified recombinant hTBP (lanes 3,4 ) or $8.7 \mu \mathrm{g}$ of $\mathrm{HeLa}$ TFIID fraction (lanes 5,6 ) were added to the reactions containing the remaining general factors from HeLa cells. Purified Gal4-Pro was added as indicated above each lane Gal4-Pro stimulates transcription with recombinant hTBP or TFIID fraction in a heat-treated nuclear extract. No TBP (lanes 1,2), $2.4 \mu \mathrm{g}$ of HeLa TFIID fraction (lanes 3,4), or 0.6 ng of recombinant hTBP (lanes 5,6) was added to a nuclear extract that had been incubated at $47^{\circ} \mathrm{C}$ for $15 \mathrm{~min}$ to inactivate the endogenous TBP activity. Gal4-Pro was added as indicated. Transcription reactions were carried out on a $\mathrm{G}_{5} \mathrm{BCAT}$ template, and the products were detected by primer extension analyses.

and TBP activities copurified (Pugh and Tjian 1990). Previous studies suggested that the coactivator activities may be present as part of a large molecular mass $>300$ $\mathrm{kD}$ ) complex, referred to as the TFIID complex, because the two activities copurify in the excluded volume of an S-300 gel filtration column. Consequently, to achieve separation of the coactivator activity from the TBP activity we have used denaturing agents such as urea at low concentrations to disrupt the TFIID complex. This procedure allowed fractionation of the endogenous TBP from its tightly associated factors while retaining tran- scriptional activity. To accomplish this we first loaded the nuclear extract from HeLa cells onto a phosphocellulose column in buffer containing $0.48 \mathrm{M} \mathrm{KCl}$ (Fig. 3A, below). Under these conditions, the TFIID complex was retained on the column while the other basal factors such as TFIIA, TFIIB, TFIIE, TFIIF, and polymerase II flowed through. The column was then eluted with buffer containing $2 \mathrm{M}$ urea and $0.45 \mathrm{M} \mathrm{KCl}$. The basal transcription activity and TBP protein (as determined by Western blot analysis) fractionated with the peak of protein eluted at this step (Fig. 2A). Interestingly, this urea- 
eluted TBP fraction retained basal transcription function but no longer responded to the addition of the Gal4-Pro activator (Fig.2B, two left-most lanes). This is in stark contrast to the TFIID fraction, obtained by standard nondenaturing methods, which is fully responsive to the Gal4-Pro activator (Fig. 1B). These results strongly suggest that urea treatment disrupted the TFIID complex bound to the column, thereby allowing the separation of TBP from its associated coactivator activity.

We then eluted the remaining proteins bound to the column by applying a 0.4 to $1 \mathrm{M}$ linear $\mathrm{KCl}$ gradient in the presence of $2 \mathrm{M}$ urea (Fig. 2A). We found that these column fractions contained no detectable basal transcription activity or TBP protein as detected by Western blot analysis (data not shown). To assay for coactivator activities, each fraction eluted by the salt gradient was added to the urea-eluted TBP fraction in the presence or absence of the Gal4-Pro activator (Fig. 2B). We identified several fractions (eluting from 0.55 to $0.65 \mathrm{M} \mathrm{KCl}$ ) that mediated the activation of transcription by Gal4-Pro. The control Gal4 protein containing the DNA-binding domain of Gal4 alone did not stimulate transcription when assayed with these coactivator fractions (data not shown). We were particularly careful to determine whether these coactivator fractions contained inhibitory activities that might depress the basal level transcription. In the absence of Gal4-Pro, the peak coactivator fractions $(0.55-0.65 \mathrm{M} \mathrm{KCl})$ had little effect on the basal level of transcription when mixed with the urea-eluted TBP fraction. In contrast, some of the lower and higher

\section{A}

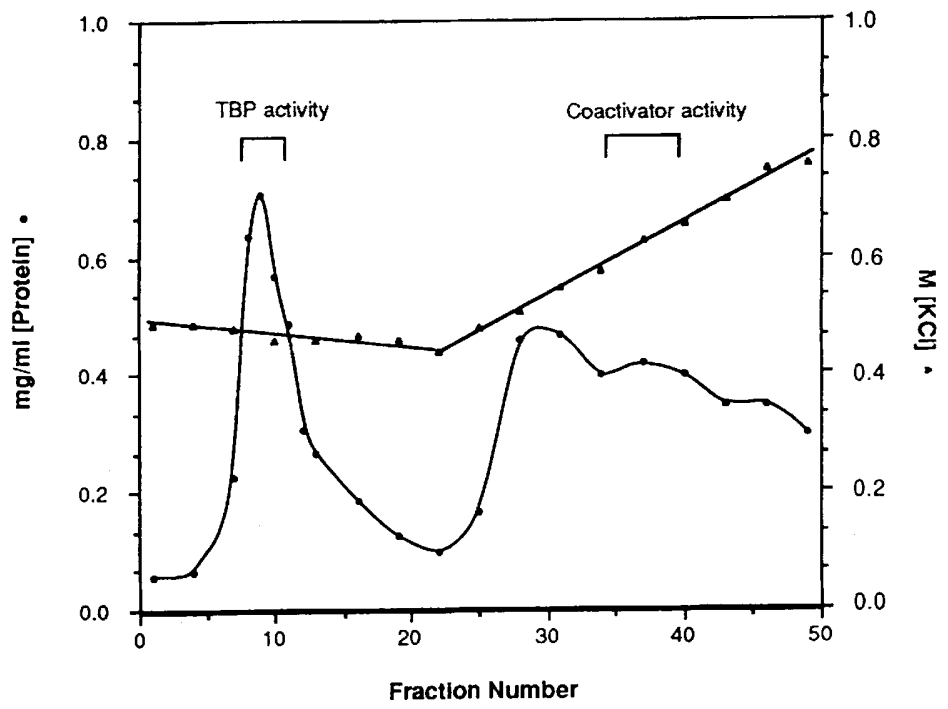

B

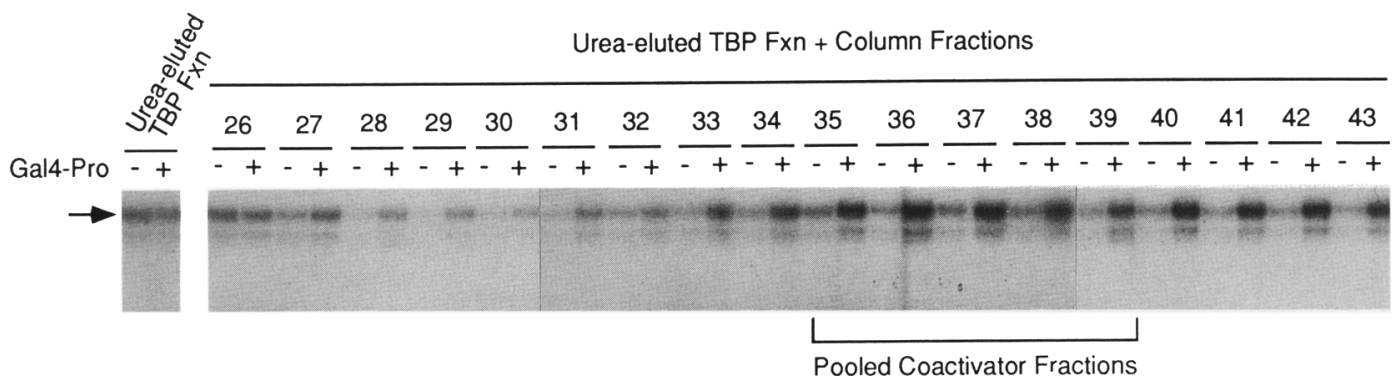

Figure 2. Chromatographic separation of TBP and coactivators in the presence of urea. $(A)$ Salt elution and total protein profile of the phosphocellulose column used to separate TBP and coactivators under denaturing conditions. Fractions that contain TBP and coactivator activities are indicated. (B) Assay for basal and activated transcription activity of proteins eluted from the column. The two left-most lanes represent endogenous TBP activity eluted from phosphocellulose with $2 \mathrm{M}$ urea and $0.45 \mathrm{M} \mathrm{KCl}$ and further purified on a heparin-agarose column (see Fig. 3A). Column fractions collected from a $\mathrm{KCl}$ gradient applied to phosphocellulose in the presence of $2 \mathrm{M}$ urea were dialyzed and directly assayed for activated transcription in reactions reconstituted with the partially purified urea-eluted TBP fraction in the presence or absence of the Gal4-Pro activator. The number above each lane corresponds to the fraction number shown in $A$. Peak coactivator fractions were pooled as indicated and were subjected to further purification as diagramed in Fig. $3 \mathrm{~A}$. 
salt fractions contained activities that repressed the basal level transcription substantially when added to the urea-eluted TBP fraction. Thus, we have achieved the separation and partial purification of coactivators for the proline activator. In addition, we have isolated a fraction containing endogenous TBP but lacking at least some of its associated coactivators.

The urea-purified TBP fraction and coactivator fraction eluted from the phosphocellulose column were subjected to further purification (Fig. 3A). The activities of these more purified fractions were tested in reconstituted transcription reactions containing the fractionated TFIIA and TFIIB/TFIIE/TFIIF/Pol II components (Fig. 3B). The urea-eluted TBP fraction and the isolated coactivators support efficient activation by the Gal4-Pro activator (lanes 5-8). However, when purified recombinant TBP was substituted for the urea-eluted TBP fraction in a reconstituted transcription assay no significant stimulation of transcription was observed with the isolated coactivators and Gal4-Pro (lanes 9,10). These results suggest that there must be some differences in the activity of the urea-eluted TBP fraction and the purified recombinant TBP. For example, it is likely that $2 \mathrm{M}$ urea disrupted the TFIID complex only partially; therefore, some components of the complex may have coeluted with TBP in the urea-eluted TBP fraction while some may have been retained on the column and eluted into the coactivator fraction. This result suggests that restoration of full coactivator function might require multiple factors. It was also apparent that conventional chromatography was not an efficient means of purifying coactivators to homogeneity. Thus, we turned to the use of alternative methods to isolate the endogenous TFIID complex and examined the properties of its components.

\section{Immunoprecipitation of the human TFIID complex reveals multiple polypeptides specifically associated with the endogenous TBP}

A powerful method of purification is the use of specific antibodies directed against TBP to isolate the complex. To affinity-purify the endogenous TFIID complex, antihuman TBP (hTBP) antibodies were incubated with the phosphocellulose $0.5-0.7 \mathrm{M} \mathrm{KCl}$ fraction in an immunoprecipitation reaction. Proteins tightly and specifically associated with TBP were subsequently eluted from the immune complex with $1 \mathrm{M}$ guanidine- $\mathrm{HCl}$ and visualized on a silver-stained gel (Fig. 4, lane 5). Under these conditions, the endogenous TBP remains largely bound to the antibody-protein A complex (lane 3). In contrast, at least six major polypeptides, as well as several other minor ones, termed TAFs (Dynlacht et al. 1991), are selectively eluted from the immune complex (lane 5). We estimate that the major TAFs are present in roughly stoichiometric amounts relative to TBP. The specificity of the immune complexes was demonstrated by using immunoaffinity resin that had been preblocked with purified recombinant TBP (lanes 6,7). At saturating amounts of recombinant TBP none of the specific TAFs could be immunopurified with the anti-TBP antibodies (lane 7), indicating that the TAFs interact specifically with the endogenous TBP. Interestingly, we found that at least 10 times more specific antibodies were required to immunoprecipitate the endogenous TBP compared with the recombinant TBP. This suggests that the majority of the epitopes on the endogenous TBP are masked by the presence of multiple polypeptides associated with the protein. Because the input shown in lane 2 represents only $1 \%$ of the total sample used to immunopurify the TAFs shown in lane 5 , we estimate that the TAFs have been purified $\sim 2000$-fold in this fraction and roughly 10,000to 20,000-fold from the crude nuclear extract. Thus, we have obtained a highly purified preparation containing a distinct set of polypeptides that are specifically associated with the native TFIID complex.

\section{The TAFs support Gal4-Pro-dependent stimulation of transcription in the presence of urea-eluted $T B P$ fraction or recombinant TBP}

We tested whether highly purified TAFs isolated by immunoprecipitation of the TFIID complex also contained coactivator activities. As expected, transcription reactions reconstituted with the urea-eluted TBP fraction that lacks coactivators failed to respond to the Gal4-Pro activator (Fig. 5A, lanes 1,2). However, addition of TAFs eluted from the immune complex restored activated transcription by Gal4-Pro (lanes 3,4), as did the control Q.2 coactivator fraction obtained by urea chromatography (lanes 5,6). Furthermore, the TAFs also stimulated transcription when incubated with the purified recombinant TBP and Gal4-Pro (lanes 7,8). Because the TAFs alone do not display any detectable transcriptional activity they appear to be free of endogenous TBP (lanes 1013). Therefore, we conclude that one or more of the TAF polypeptides specifically immunopurified from the TFIID complex is sufficient to restore coactivator functions for the proline-rich activator.

It has been suggested in a previous study that the coactivator-TBP interactions are species specific (Pugh and Tjian 1990). Therefore, we tested whether the isolated human TAF polypeptides restore activated transcription with urea-eluted hTBP but not Drosophila TBP. A chimeric activator Gal4-Gln, bearing the activation domain of $\mathrm{Spl}$, was used in this assay because $\mathrm{Spl}$ has been shown to stimulate transcription in a reaction reconstituted with the general factors from HeLa cells and the TFIID fraction from Drosophila (Hoey et al. 1990). We found that neither the immunopurified human TAFs nor the Q.2 coactivator fraction supported activated transcription with urea-eluted Drosophila TBP and Gal4 Gln (Fig. 5B, lanes 3-6), whereas the same fractions stimulated transcription efficiently in the control reactions with urea-eluted TBP fraction from HeLa cells (lanes $7-10$ ). These results lend further support to the notion that the coactivators may function in a species-specific manner, presumably interacting, at least in part, with the unique amino-terminal domain of TBP. 
A
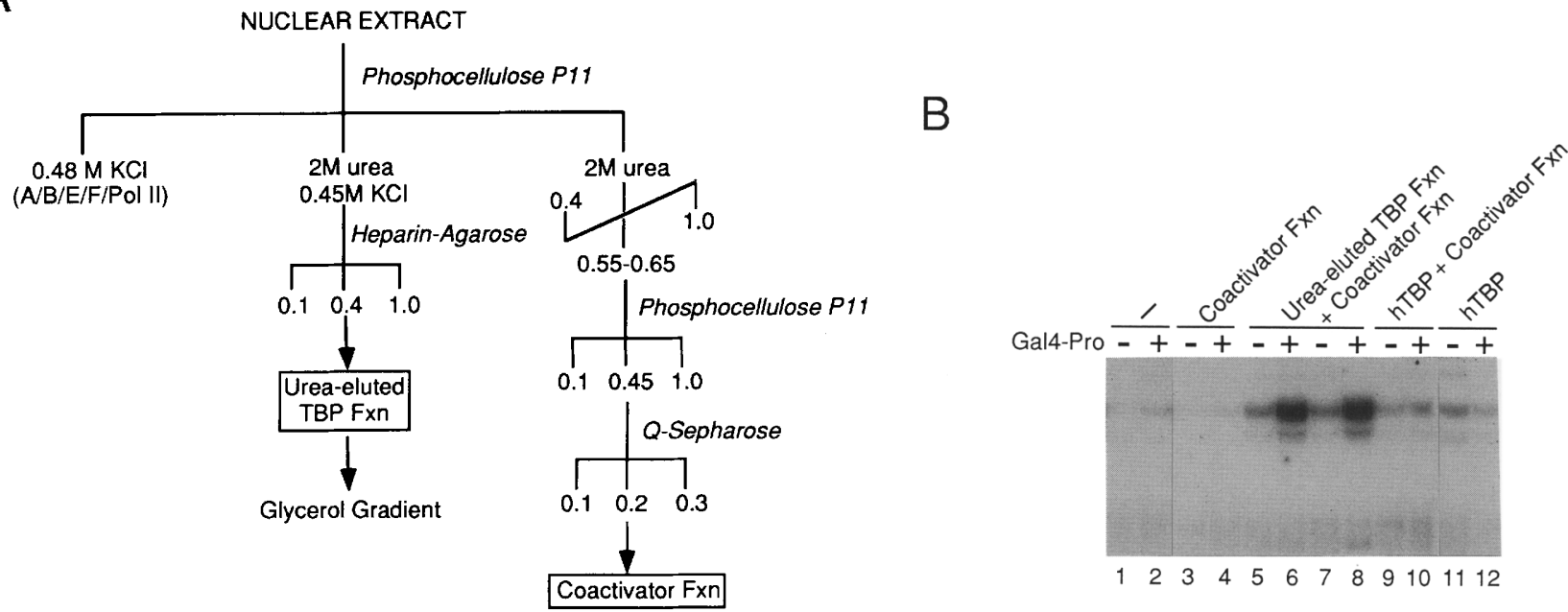

Figure 3. Further purification of urea-eluted TBP and coactivator fractions. $(A)$ Diagram depicting the purification scheme; $(B)$ transcriptional activity of the coactivator fraction. Reactions were reconstituted on $\mathrm{G}_{5} \mathrm{E} 4 \mathrm{~T}$ template with partially purified TFIIA, and TFIIB/TFIIE/TFIIF/Pol II fractions in the presence $(+)$ or absence $(-)$ of Gal4-Pro as indicated. No TBP (lanes 1,2), 0.35 $\mu \mathrm{g}$ of coactivator fraction (lanes 3,4), $0.4 \mu \mathrm{g}$ of urea-eluted TBP fraction plus $0.1 \mu \mathrm{g}$ (lanes 5,6$)$ or $0.35 \mu \mathrm{g}(\operatorname{lanes} 7,8)$ of coactivator fraction, $0.4 \mathrm{ng}$ of recombinant hTBP plus $0.35 \mu \mathrm{g}$ of coactivator fraction (lanes 9,10), and $0.4 \mathrm{ng}$ of recombinant hTBP (lanes 11,12) were added to the reaction.

$T B P$ in the urea-eluted TBP fraction is complexed with a subset of TAFs

As discussed earlier, we have found that the coactivator fraction obtained by phosphocellulose column chromatography in the presence of urea is capable of restoring activated transcription with the urea-eluted TBP fraction but not with the recombinant TBP (Fig. 3B). To examine these potential differences more carefully we performed glycerol gradient sedimentation analyses of the native TFIID complex, urea-eluted TBP fraction, and recombinant TBP (Fig. 6). As predicted, the peak of TBP in the endogenous native TFIID fraction sedimented to the bottom of the glycerol gradient as a large complex with a molecular mass of $>300 \mathrm{kD}$, whereas the recombinant TBP sedimented as a monomer of $40 \mathrm{kD}$ (Fig. 6A). In contrast, TBP in the urea-eluted TBP fraction distributed over a broad range of sedimentation values ranging from 300 to $100 \mathrm{kD}$ across the gradient, suggesting the presence of multimeric complexes (Fig. 6B). Because addition of urea to the glycerol gradient converted TBP in this fraction to sediment like a monomer (Fig. $6 \mathrm{C}$ ) it is possible that in the urea-eluted TBP fraction, removal of urea by dialysis allowed the endogenous TBP to partially reassemble into a complex with other TBP-associated proteins that cofractionated with it. When TBP in the urea-eluted TBP fraction was immunoprecipitated with anti-TBP antibodies we detected a subset of TAFs that are normally eluted from the native TFIID complex in the presence of guanidine- $\mathrm{HCl}$ (Fig. 6D, cf. lanes 1 and 2). These experiments suggest that TBP in the urea-eluted TBP fraction is complexed with a subset of TAFs that may be required for efficient interaction between the components in the coactivator fraction and TBP to stim- ulate transcription. Furthermore, additional TAFs that are missing from the urea-eluted TBP fraction must be important for coactivator function. Therefore, at least two different coactivator components appear to participate in mediating Gal4-Pro-dependent stimulation of transcription.

The coactivator fraction is capable of supporting transcriptional activation by the prolineand glutamine-rich activators but not the VP16 activator protein

We wished to determine whether the isolated coactivator fraction is capable of responding to activators bearing different types of activation domains. We therefore compared the activation properties of chimeric proteins bearing the DNA-binding domain of Gal4 fused to the activation domain of either Sp1 (Gal4-Gln), VP16 (Gal4VP16), or CTF (Gal4-Pro) so that their transcriptional activities could be conveniently assayed with the same template DNA. As expected, the addition of Gal4-Pro, Gal4-Gln, and Gal4-VP16 to reconstituted reactions containing the urea-eluted TBP fraction without the coactivators does not alter the basal level of transcription significantly (Fig. 7, lanes 1-4). The coactivator fraction in the absence of TBP gives no detectable transcription whether or not any of the activators are present in the reaction (lanes 9-12). However, in the presence of the urea-eluted TBP fraction and the coactivator fraction, both Gal4-Pro and Gal4-Gln activators restored activated transcription (lanes 5-7). In contrast, Gal4-VP16 failed to stimulate transcription under these conditions 

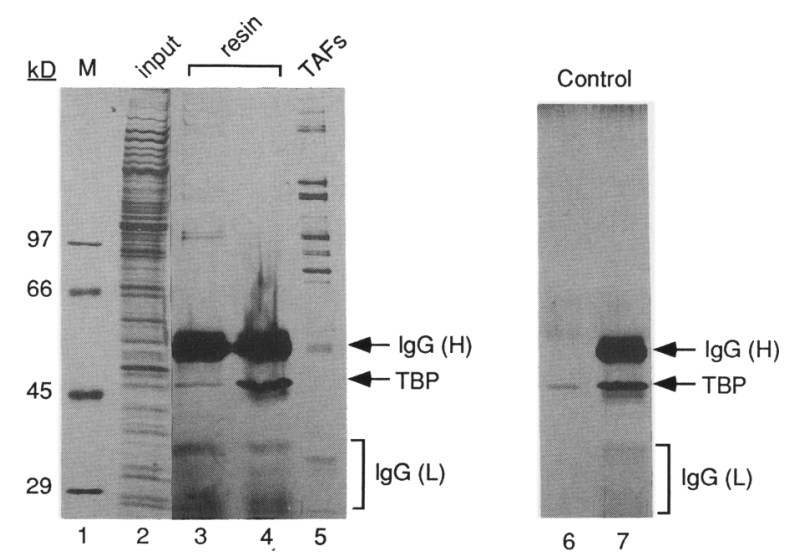

Figure 4. Immunopurification of human TFID complex indicates that endogenous TBP is associated with a distinct set of polypeptides. Phosphocellulose $0.5-0.7 \mathrm{M} \mathrm{KCl}$ fraction (input, lane 2) was incubated with affinity-purified anti-TBP antibodies to immunoprecipitate the native TFIID complex. Purified immune complexes were treated with $1 \mathrm{M}$ guanidine- $\mathrm{HCl}$, and the TAF polypeptides released into the supernatant under these conditions are shown in lane 5 . The pellet containing the TBPantibody complex bound to the resin after elution of TAFs is shown in lane 3. As controls, immunoprecipitation reactions were carried out in parallel by using the same input fraction with antibodies that had been preblocked with recombinant TBP. The supernatant obtained following the guanidine- $\mathrm{HCl}$ elution of these purified immune complexes is shown in lane 6 , and the pellet is shown in lanes 4 and 7 . (lane 8 ) or in the presence of the partially purified but intact TFIID fraction (lane 16) even though Gal4-VP16 activates transcription in a crude nuclear extract (lanes $17,20)$. These results suggest that proline and glutamine activators can utilize coactivators present in the same

fraction derived from the urea-treated TFIID. At this point, we do not know whether the two activators use the same or different coactivators because individual coactivators have not been purified to homogeneity. In contrast, the Gal4-VP16 activator clearly failed to enhance transcription under these same conditions. Therefore, we propose that the cofactor requirements for activation by Gal4-VP16 differ significantly from those of proline- or glutamine-rich activators and that these different classes of activators may work via distinct mechanisms.

\section{Discussion}

\section{Biochemical isolation of coactivators that mediate transcriptional activation}

Previous work from this laboratory led to the hypothesis that a novel class of transcription factors termed coactivators are required to mediate activation of transcription by certain promoter selective regulators in animal cells. In this paper we demonstrate that coactivators are tightly associated with the human TBP and that they can be isolated chromatographically from the TFIID fraction

A

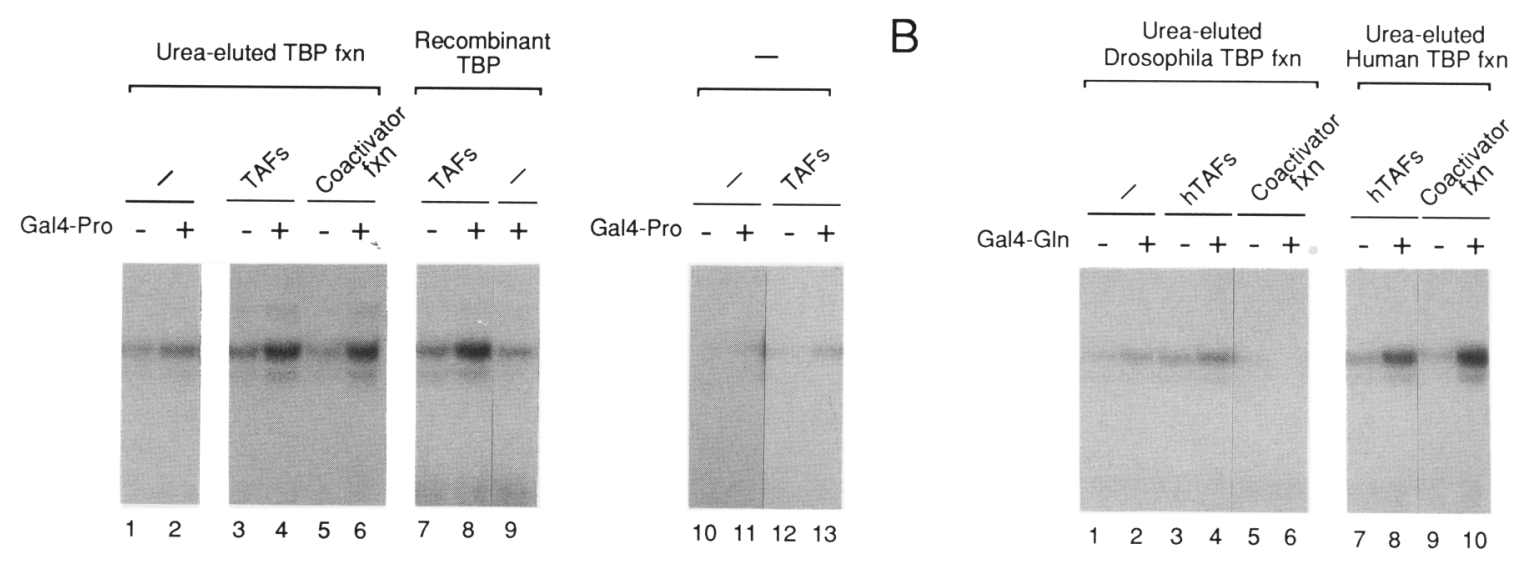

Figure 5. Transcriptional activity of TAFs. (A) TAFs mediate Gal4-Pro-dependent transcription in the presence of urea-eluted TBP fraction or recombinant TBP. Transcription reactions were reconstituted on a $\mathrm{G}_{5} \mathrm{E} 4 \mathrm{~T}$ template with general factors and additional factors as indicated above each lane. Reactions contained $1 \mu \mathrm{g}$ of partially purified urea-eluted TBP fraction (lanes 1-6) or 0.8 ng of recombinant TBP (lanes 7-9). TAFs were assayed for activated transcription in the presence of Gal4-Pro and urea-eluted TBP fraction (lanes 3,4 ) or recombinant TBP (lanes 7,8). Reactions in lanes 3 and 4 and 7 and 8 contained $\sim 50$ and 100 ng of TAFs, respectively. Control reactions were carried out with the Q.2 coactivator fraction and urea-eluted TBP fraction $($ lanes 5,6$)$. TAFs alone did not have any detectable transcriptional activity (lanes 10-13). (B) Human TAFs restore activated transcription with urea-eluted hTBP fraction but not Drosophila TBP fraction. Transcription reactions contained $1.5 \mu \mathrm{g}$ of urea-eluted Drosophila TBP fraction (lanes 1-6) or 1 $\mu \mathrm{g}$ of urea-eluted hTBP fraction (lanes 7-10). Reactions were carried out in the presence of $\sim 50$ ng of human TAFs (lanes $3,4,7,8)$ or 0.35 $\mu \mathrm{g}$ of Q.2 coactivator fraction (lanes 5,6,9,10). A chimeric activator Gal4-Gln bearing the glutamine-rich activation domains of Sp1 was added as indicated above each lane. The source of urea-eluted Drosophila TBP fraction was the $0.18 \mathrm{M} \mathrm{KCl} \mathrm{plus} 3 \mathrm{M}$ urea fraction from the Q-Sepharose/urea column (Dynlacht et al. 1991). 
following disruption of the TFIID complex with denaturing agents. In addition, we have purified the native TFIID complex by immunoaffinity resin coupled to anti-human TBP antibodies and shown that polypeptides specifically bound to the endogenous TBP are responsible for coactivator activity. Our results suggest that transcriptional regulators bearing proline-rich activation domains function through these coactivators to stimulate transcription. Figure 8 depicts the most parsimonious interactions that are likely to occur between an activator (Gal4-Pro) and its targeting components in the basal transcriptional machinery. This diagram is an extension of our previous model (Pugh and Tjian 1990) illustrating potential mechanisms of action by coactivators. We have drawn the TFIID complex as consisting of TBP and multiple TAFs, including coactivators that are responsible for mediating the activation of DNA-bound transcriptional regulators such as the Gal4-Pro activator. Although we hypothesize that activators such as Gal4-Pro and Spl interact directly with coactivators which, in turn, act as intermediary targets leading to contact with TBP and the other basal factors, there is no direct biochemical or functional evidence to determine the nature of these interactions. We think that not all TAFs need be coactivators that interact directly with TBP, but it seems clear that at least some of the TAFs must bind directly to TBP. In gel mobility-shift assays with recombinant TBP we detect distinct slower-migrating protein-DNA complexes that contain TBP when the isolated coactivators or TAFs are present in the reaction, confirming that some coactivator component(s) can interact directly with TBP (data not shown). It is also possible that one of the TAFs might be TFIIA, as previous studies have demonstrated that TFIIA and TFIID interact to form a complex in a gel-shift assay (Buratowski et al. 1989; Maldonado et al. 1990).

An independent study conducted recently with Drosophila embryo extracts confirms many of the properties ascribed to coactivators described here (Dynlacht et al. 1991). For example, the Drosophila transcription study reveals that a specific set of polypeptides can be immunoprecipitated with antibodies directed against the recombinant Drosophila TBP. The polypeptides eluted from these immunoprecipitated complexes by urea also have coactivator activities because they are necessary for restoring activation by the developmentally regulated transcription factor NTF-1. Thus, in both human and Drosophila cells, coactivators appear to be tightly associated with TBP and play a critical function in the assembly of an activated initiation complex. Taken together, these results strongly indicate that the multisubunit, multifunctional nature of the TFIID complex is a general property, at least in higher eukaryotes, and the notion that the single subunit TBP and TFIID fraction carry out identical functions is no longer tenable. Comparison of the human and Drosophila TAFs reveals some similarities in their pattern of polypeptides, but most TAFs differ in size between the two species. Also, more polypeptides appear to be associated with the hTBP than the Drosophila TBP. It is possible that the structure of human TFIID is more complex than its counterpart in Drosophila. We have also found that the human coactivator fraction and TAFs fail to restore activated transcription with urea-eluted Drosophila TBP, suggesting that the coactivators may function in a species-specific manner as proposed in our previous study (Pugh and Tjian 1990).

Various laboratories have studied the mechanism of activation by acidic activators. It has been reported that Gal4-VP16 interacts directly with TBP (Stringer et al. 1990) or TFIIB (Lin and Green 1991) to activate transcription. However, others suggest that these activators require a target factor (termed mediator or adaptor) distinct from the general factors (Berger et al. 1990; Kelleher et al. 1990; Martin et al. 1990). A recent report describes the isolation of a mediator fraction from yeast that is required for activation by acidic activators in vitro (Flanagan et al. 1991). Although the mediator fraction restores activation by the acidic activators with the recombinant TBP, it is likely to contain a different type of transcription factor from the coactivator fraction or TAFs described in this paper. In yeast, the TBP apparently exists as a monomer without any tightly associated factors $(\mathrm{Bu}-$ ratowski et al. 1988; Cavallini et al. 1988; Horikoshi et al. 1989b). Thus, the isolated yeast mediator is presumably free of TBP in its native form while the coactivators and TAFs from HeLa cells are tightly complexed with the endogenous TBP. Therefore, the yeast mediator need not be an intermediary between the activator and TBP.

Interestingly, under our assay conditions we have failed to observe activation by Gal4-VP16 with the coactivator fraction or even with the partially purified TFIID fraction (Fig. 7). These results suggest that Gal4VP16 mediates activation by a different mechanism that requires factors not found in the reconstituted reaction used in this study. Alternatively, it is possible that Gal4 VP16 enhances transcription largely by allowing basal factors to exclude nucleosomes or histones from occupying the promoter region (Croston et al. 1991; Workman et al. 1991). Perhaps the reason we have failed to observe an efficient stimulatory effect by Gal4-VP16 is because our in vitro reactions contain very little histone or other inhibitors of the DNA template.

\section{The human TFIID complex consists of TBP, coactivators, and other tightly associated polypeptides required for efficient activation of transcription}

In reconstituted transcription assays we have found that the urea-eluted TBP fraction restores activated transcription efficiently with the coactivator fraction, but the recombinant TBP responds poorly. Glycerol gradient sedimentation studies suggest that other proteins are complexed with the endogenous TBP in the urea-eluted TBP fraction. Immunoprecipitation of the TBP-containing complex from the urea-eluted TBP fraction demonstrates directly that the TBP in this fraction remains associated with a subset of TAFs. These results suggest a function for additional TAFs that are bound to TBP in mediating 
A

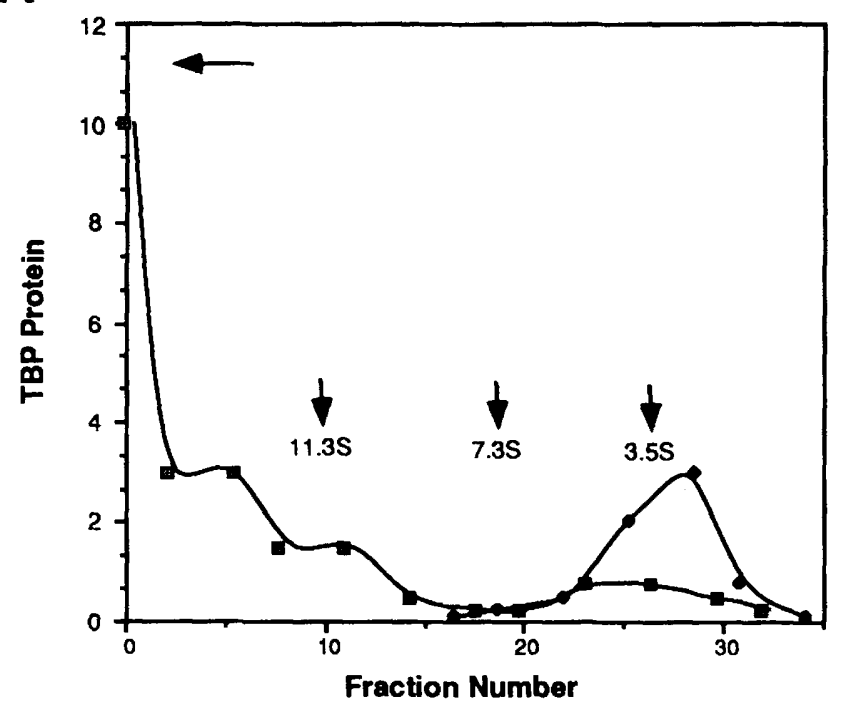

B

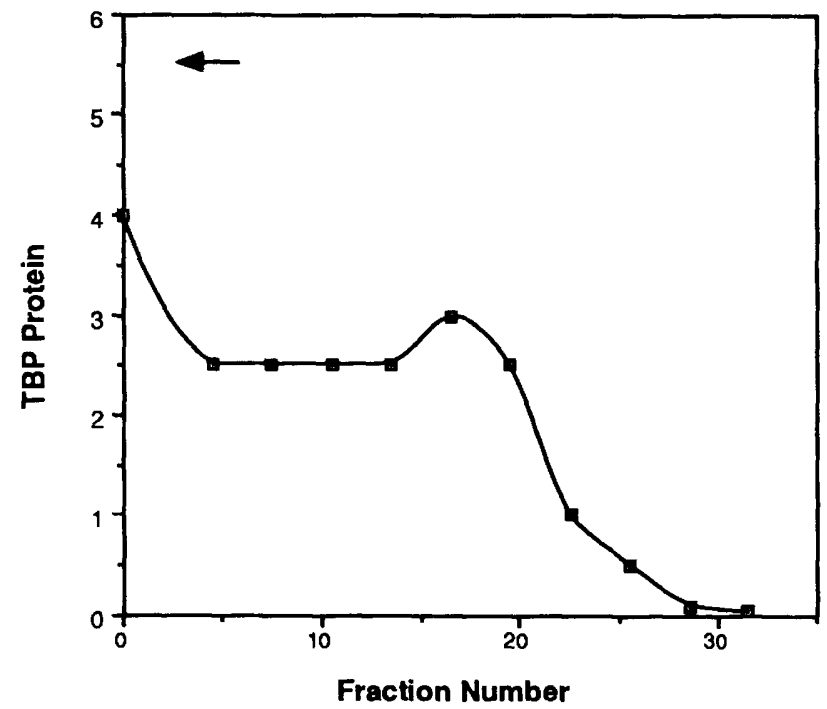

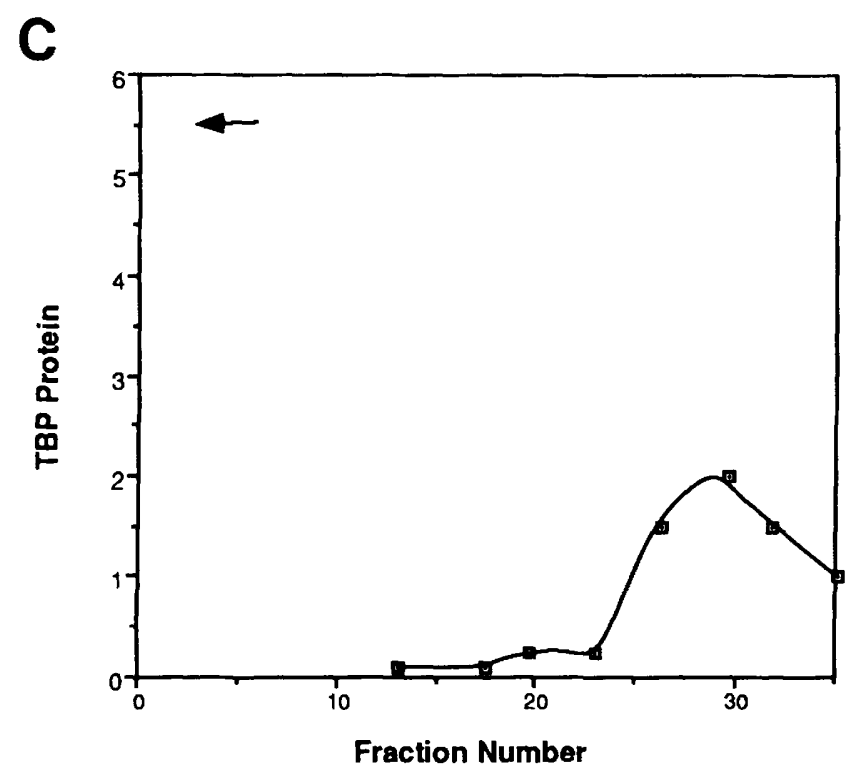

Figure 6. TBP in urea-eluted TBP fraction is associated with a subset of TAFs. $(A-C)$ Glycerol gradient sedimentation analyses of various preparations of TBP. The graphs show approximate amounts of TBP (in arbitrary units) present in fractions from glycerol gradients, as estimated by visual inspection of Western blots. Arrows indicate the direction of sedimentation. (A) Native TFIID fraction (phosphocellulose 0.5-0.7 M KCl eluate) (squares) and recombinant TBP (diamonds) were run in parallel gradients. Positions for three molecular mass standards (catalase, $232 \mathrm{kD}$; aldolase, $158 \mathrm{kD}$; ovalbumin, $45 \mathrm{kD}$ ) are also shown. (B) Urea-eluted TBP fraction. $(C)$ Urea-eluted TBP fraction analyzed in a gradient containing urea. (D) Comparison of TAFs present in the phosphocellulose 0.5-0.7 $\mathrm{M}$ $\mathrm{KCl}$ TFIID fraction (lane 2) and TBP fraction obtained from urea chromatography (lane 1). Immunoprecipitation reactions were carried out as described in Fig. 4, and polypeptides eluted from the immune complexes in the presence of $1 \mathrm{M}$ guanidine- $\mathrm{HCl}$ are shown in lanes 1 and 2; the corresponding pellet after such treatment is shown in lanes 3 and 4 , respectively. The TFIID fraction used in this experiment differed slightly from the TFIID fraction shown in Fig. 4 (prepared by standard methods) in that it was obtained by loading a crude nuclear extract onto a phosphocellulose column at $0.48 \mathrm{M} \mathrm{KCl}$ (instead of $0.1 \mathrm{M} \mathrm{KCl}$ ) and eluting at $0.7 \mathrm{M} \mathrm{KCl}$, in parallel to the column eluted in the presence of urea.

efficient interaction between the coactivators and TBP. In the absence of these additional TAFs the recombinant TBP may be incapable of forming a productive TFIID complex with the components of the coactivator fraction. Thus, some or all of the TAFs may have coactivator function. Because TFIID is a very large multicomponent complex, it is also possible that some TAFs might function similarly to chaperone proteins that assist in the assembly of multisubunit protein complexes (Ellis and Hemmingsen 1989|. 


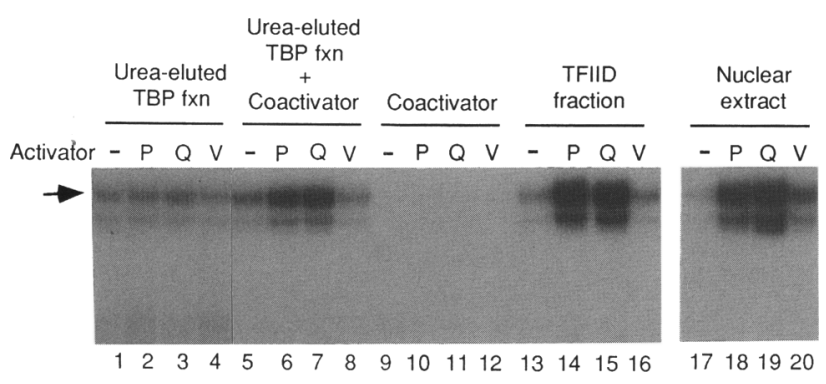

Figure 7. Gal4 derivatives bearing different types of activation domains differ in their ability to stimulate transcription with coactivators. Transcription reactions were reconstituted on $\mathrm{G}_{5} \mathrm{E} 4 \mathrm{~T}$ template with general factors from HeLa cells (lanes 1-16). Partially purified urea-eluted TBP fraction $(1.6 \mu \mathrm{g})$, coactivator $(0.35 \mu \mathrm{g})$, and native TFIID $(12 \mu \mathrm{g})$ fractions were added to the reaction as indicated (top). Reactions carried out in a crude nuclear extract are shown in lanes 17-20. Approximately 1 footprint unit of each activator protein-Gal4-Pro (P), Gal4Gln (Q), Gal4-VP16 (V)-was added to the reactions as indicated above each lane.

Why are so many proteins involved in mediating transcriptional activation? It appears that transcriptional activators possess different types of activation domains that presumably contact distinct target proteins to exert their effects on transcription. If the TBP is the only target of all activators, it is not easy to explain how such a small protein can simultaneously contact multiple activating regions. Thus, it seems more reasonable to propose that coactivators and other proteins associated with TBP provide additional protein surfaces to accommodate multiple protein-protein interactions with a diverse array of transcriptional regulators. It is interesting to note that some of the TAFs are present in apparent substoichiometric amounts in the immunopurified TBP complexes. This is what one might expect if different sets of coactivators/TAFs are associated with different TFIID complexes, thus generating unique and specific interaction surfaces as targets for different activators. In addition, coactivators, like activators, might be regulated post-translationally or by developmental/tissue-specific programs of expression, thus serving as an additional control point in transcription initiation. Recent studies demonstrating the involvement of TBP in transcription by RNA polymerase III (Margottin et al. 1991; Simmen et al. 1991) suggest further that TBP may be associated with a wide variety of polypeptides to form multiple complexes that participate in transcription by more than one RNA polymerase. It will be of interest to investigate whether or not a TFIID complex consisting of TBP and a unique set of TAFs is also involved in transcription by RNA polymerase I. Further purification and characterization of the individual components of the TFIID complex should help us to understand the mechanisms by which sequence-specific transcription factors interact with TBP and the general factors to regulate transcription.

\section{Materials and methods}

Template DNAs

Template DNAs containing a TATA box from the adenovirus E4 promoter, with or without the five upstream Gal4-binding sites $\left(\mathrm{G}_{5} \mathrm{E} 4 \mathrm{~T}\right.$ and $\mathrm{E} 4 \mathrm{~T}$, respectively), have been described (Lin et al. 1988). The $\mathrm{G}_{5} \mathrm{BCAT}$ template used in some transcription assays contained a TATA box from the adenovirus E1B promoter and five upstream Gal4-binding sites as described (Lillie and Green 1989).

\section{Expression and purification of Gal4 fusion proteins}

To construct the plasmid pG3 that directs the expression of amino acids 1-94 of Gal4 in Escherichia coli, the XhoI-XbaI fragment of pTMCl (Carey et al. 1989) was first replaced with the Xhol-Xbal fragment of pSG424 (Sadowski and Ptashne 1989) to generate pbGl. Plasmid pbGl was then digested with Sall, and ends were filled in with the Klenow fragment of DNA polymerase I and digested with $\mathrm{HpaI}$ to remove sequences encoding amino acids 95-147 to generate pG3. Removal of these sequences was necessary because they contain acidic residues that stimulate transcription under the in vitro reaction conditions used (M. Carey, pers. comm.). Dimerization and DNAbinding properties of Gal4 are retained in the truncated Gal4 (1-94) protein. Plasmid pP13 expressing Gal4-Pro fusion protein was constructed by inserting the BgIII-HindIII fragment of pCTF1 (Santoro et al. 1988) corresponding to the DNA sequences encoding the carboxy-terminal 100 amino acids of pCTFl into plasmid pG3 digested with $\mathrm{XbaI}$ and $S \mathrm{maI}$. All ends were filled in with the Klenow fragment of DNA polymerase I prior to ligation. The plasmid expressing Gal4-Gln fusion protein was constructed by inserting the DNA sequences encoding amino acids $82-500$ of $S p 1$ that include activation domains $A$ and B (Courey and Tjian 1988) into the SmaI site of pG3. The Gal4 derivatives were expressed in E. coli strain XA-90, and the proteins were purified from the soluble fraction by DNA affinity chromatography as described (Baichwal and Tjian 1990). The plasmid expressing Gal4-VP16 has been described, and the recombinant protein was purified according to published procedures (Chasman et al. 1989).

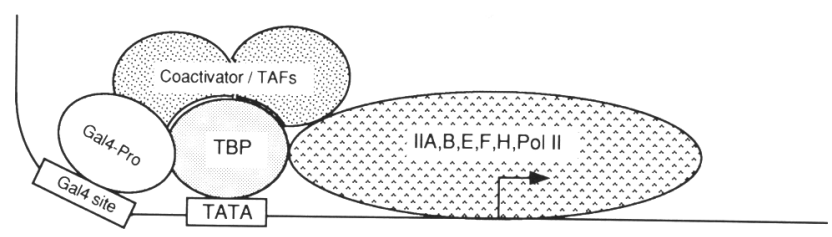

Figure 8. A model for transcriptional activation mediated by coactivators and other TBP-associated proteins. This diagram illustrates a potential interplay among DNA, activator, coactivators/TAFs, TBP, and the transcription apparatus. The precise mechanism of action and the specific contacts between factors have not been determined; therefore, only the simplest possible model consistent with our data is depicted. The model is similar to the one described previously by Pugh and Tiian (1990), which proposes that coactivators serve as intermediaries that connect the activator (Gal4-Pro) to TBP and the general transcription machinery. The results presented in this paper suggest that TBP and multiple TAF polypeptides that include coactivators are required for efficient interaction between activators and general factors to activate transcription. 


\section{In vitro transcription}

Transcription assays were performed essentially as described (Pugh and Tjian 1990), and the products were detected by primer extension analysis. In most cases, transcription reaction was reconstituted on template DNA (200 ng) with the general factors $(24 \mu \mathrm{g})$ in the flowthrough fraction (P.48) from a phosphocellulose P1I column obtained after loading a crude HeLa nuclear extract onto the column in PC buffer $20 \mathrm{mM}$ HEPES- $\mathrm{KOH}$ at $\mathrm{pH} 7.9,2 \mathrm{~mm} \mathrm{MgCl}, 10 \mu \mathrm{m} \mathrm{ZnSO}, 1 \mathrm{~mm}$ DTT, $10 \%$ glycerol) containing $0.48 \mathrm{M} \mathrm{KCl}$. The column was subsequently washed and eluted in PC buffer containing $0.7 \mathrm{M} \mathrm{KCl}$ to obtain the native TFIID fraction (P.7). The P.48 fraction, as well as all the fractions used in transcription reactions, was dialyzed against $0.1 \mathrm{M}$ HEMG buffer $(25 \mathrm{mM}$ HEPES- $\mathrm{KOH}$ at $\mathrm{pH} 7.9,12.5$ $\mathrm{mM} \mathrm{MgCl}_{2}, 0.1 \mathrm{~mm}$ EDTA, 20\% glycerol, $0.1 \% \mathrm{NP}-40,1 \mathrm{~mm}$ DTT). Recombinant hTBP used in most assays was purified from $\mathrm{HeLa}$ cells infected with a recombinant vaccinia virus expressing hTBP as described previously (Peterson et al. 1990). In some cases, reconstituted transcription was also performed with partially purified TFIIA and TFIIB/TFIIE/TFIIF/Pol II fractions as described (Pugh and Tjian 1990). Transcription assays in crude nuclear extracts or in extracts treated with heat were carried out as described (Pugh and Tjian 1990). Heat-treated extract was prepared by incubating a crude nuclear extract at $47^{\circ} \mathrm{C}$ for $15 \mathrm{~min}$ to inactivate the endogenous TBP activity $(\mathrm{Na}$ kajima et al. 1988). Approximately 1 footprint unit (minimal amount of protein required to completely protect the Gal4binding site in a footprinting assay) of activator protein was used in all assays.

\section{Fractionation of TFIID in urea}

Nuclear extract from 50 liters of HeLa spinner cells $\left(2.5 \times 10^{10}\right.$ cells) was prepared as described (Pugh and Tjian 1990) and loaded on to a phosphocellulose P11 column in PC buffer (see above) containing $0.48 \mathrm{M} \mathrm{KCl}$ and washed in the same buffer. The bound fraction was first eluted with 1.4 column volumes of HEMG buffer containing $0.45 \mathrm{M} \mathrm{KCl}$ and $2 \mathrm{M}$ deionized urea. Proteins were then eluted by applying a $0.4-1.0 \mathrm{M}$ linear $\mathrm{KCl}$ gradient in PC buffer (5 column volumes) in the presence of $2 \mathrm{M}$ urea. The fractions collected were dialyzed immediately against $0.1 \mathrm{M}$ HEMG buffer and assayed for basal and activated transcription in reconstituted reactions with P.48 fraction on $G_{5} E 4 T$ template. The assay for coactivator activity was performed similarly except $1.6 \mu \mathrm{g}$ of partially purified urea-eluted TBP fraction (heparin-agarose $0.4 \mathrm{M}$ fraction in Fig. $3 \mathrm{~A}$ ) was added to each reaction. To further purify the TBP fraction eluted from phosphocellulose column with urea, dialyzed fraction (in $0.1 \mathrm{M}$ HEMG) was loaded on a heparin-agarose column in the same buffer. TBP activity was recovered in $0.4 \mathrm{M} \mathrm{KCl}$ eluate, dialyzed against $0.1 \mathrm{M} \mathrm{HEMG}$, and used in subsequent experiments. Phosphocellulose column fractions containing the coactivator activity $(0.55-0.65 \mathrm{KCl}$ plus $2 \mathrm{M}$ urea eluate) were pooled, dialyzed, and loaded onto a second phosphocellulose column in PC buffer containing $0.1 \mathrm{M} \mathrm{KCl}$. The activity, recovered in $0.45 \mathrm{M}$ $\mathrm{KCl}$ eluate, was diluted to $0.1 \mathrm{M} \mathrm{KCl}$ in $\mathrm{HEMG}$ and loaded onto a Q-sepharose column equilibrated with $0.1 \mathrm{M}$ HEMG. The $0.2 \mathrm{M} \mathrm{KCl}$ eluate containing the coactivator activity was dialyzed against 0.1 M HEMG and used in all experiments (fold purification estimated at 300-500).

\section{Immunoprecipitation reactions}

Polyclonal antisera raised against bacterially produced recom- binant hTBP were purified by using TBP-coupled affinity resins. Immunoprecipitation reactions were carried out with a procedure similar to the one that has been described (Dynlacht et al. 1991). Approximately $400 \mu \mathrm{g}$ of the phosphocellulose $0.5-0.7 \mathrm{M}$ $\mathrm{KCl}$ fraction (TFIID fraction) was incubated with $4 \mu \mathrm{g}$ of affinity-purified anti-hTBP antibodies at $4^{\circ} \mathrm{C}$ on a rotating wheel for several hours. Protein A-Sepharose (Pharmacia) was then added, and the mixture was incubated at $4^{\circ} \mathrm{C}$ overnight. The antibody-protein A complexes were pelleted in an Eppendorf microcentrifuge (2000 rpm) and washed four times with PC buffer. TAFs were eluted from the complexes in Gl buffer 175 mM potassium glutamate, $15 \mathrm{~mm}$ Tris-acetate at $\mathrm{pH} 7.9,15 \%$ glycerol, $1.5 \mathrm{mM} \mathrm{MgCl}_{2}, 0.1 \% \mathrm{NP}-40,0.15 \mathrm{~mm}$ EDTA, $1.75 \mathrm{~mm}$ $\mathrm{DTT}, 100 \mu \mathrm{g} / \mathrm{ml}$ of insulin, and $1 \mathrm{M}$ guanidine- $\mathrm{HCl}$ ), precipitated with trichloroacetic acid (TCA), and analyzed on SDSPAGE. The resin left behind after elution of TAFs was resuspended in protein sample buffer and loaded onto SDS gels. For transcription studies the eluted TAFs were dialyzed in transcription buffer. Control immunoprecipitation reactions were performed in parallel using antibodies $(4 \mu \mathrm{g})$ that had been incubated with $800 \mathrm{ng}$ of purified recombinant TBP from $E$. coli for $3 \mathrm{hr}$ on ice prior to incubation with the TFIID fraction.

\section{Glycerol gradient sedimentation analysis}

Protein $(50-100 \mu \mathrm{l})$ was applied to $2-\mathrm{ml}$ linear glycerol gradients $(10-30 \%)$ with HEMG containing $0.1 \mathrm{M} \mathrm{KCl}$, except $2.4 \mathrm{M}$ deionized urea was added to gradient $C$. Gradients were centrifuged at $50,000 \mathrm{rpm}$ for $8 \mathrm{hr}$ in a Beckman TLS 55 rotor. Thirty-four fractions of $50 \mu \mathrm{l}$ each were collected, and proteins were precipitated with TCA. Samples were analyzed by SDS-PAGE, followed by Western blot analysis with affinity-purified rabbit polyclonal antibodies raised against recombinant hTBP.

\section{Acknowledgments}

We thank N. Mermod for conducting the initial in vivo studies with Gal4-Pro fusions, G. Peterson for providing Gal4-Gln and Gal4-VP16 fusion proteins and anti-hTBP antiserum, and V.J.Baichwal for Gal4 affinity resin. We are indebted to B. Johnson, L. Bradley, and A. Park for supplying us with large amounts of HeLa nuclei. We especially thank A. Admon, S. Bell, M. Botchan, B. Dynlacht, G. Gill, T. Hoey, G. Peterson, and members of the Tjian laboratory for valuable discussions throughout this project and helpful comments on the manuscript. This investigation was supported in part by a grant from the National Institutes of Health (to R.T.), an American Cancer Society, California Division fellowship (S-60-90 to N.T.), and a Leukemia Society of America fellowship (to B.F.P.).

The publication costs of this article were defrayed in part by payment of page charges. This article must therefore be hereby marked "advertisement" in accordance with 18 USC section 1734 solely to indicate this fact.

\section{References}

Baichwal, V.R. and R. Tjian. 1990. Control of c-jun activity by interaction of a cell-specific inhibitor with regulatory domain $\delta$ : Differences between v- and c-jun. Cell 63: 815-825.

Berger, S.L., W.D. Cress, A. Cress, S.J. Triezenberg, and L. Guarente. 1990. Selective inhibition of activated but not basal transcription by the acidic activation domain of VP16: Evidence for transcriptional adaptors. Cell 61: 1199-1208. Buratowski, S., S. Hahn, and A. Sharp. 1988. Function of a yeast 
TATA element binding protein in a mammalian transcription system. Nature 334: 37-42.

Buratowski, S., S. Hahn, L. Guarente, and P.A. Sharp. 1989. Five intermediate complexes in transcription initiation by RNA polymerase II. Cell 56: 549-561.

Carey, M., H. Kakidani, J. Leatherwood, F. Mostashari, and M. Ptashne. 1989. An amino terminal fragment of GAL4 binds DNA as a dimer. I. Mol. Biol. 209: 423-432.

Cavallini, B., J. Huet, J. Plassat, A. Sentenac, J. Egly, and P. Chambon. 1988. A yeast activity can substitute for the HeLa cell TATA box factor. Nature 334: 77-80.

Cavallini, B., I. Faus, H. Matthes, J.M. Chipoulet, B. Winsor, J.M. Egly, and P. Chambon. 1989. Cloning of the gene encoding the yeast protein BTF1Y, which can substitute for the human TATA box-binding factor. Proc. Natl. Acad. Sci. 86: 9803-9807.

Chasman, D.I., J. Leatherwood, M. Carey, M. Ptashne, and R.D. Kornberg. 1989. Activation of yeast polymerase II transcription by Herpesvirus VP16 and GAL4 derivatives in vitro. Mol. Cell. Biol. 9: 4746-4749.

Courey, A.J. and R. Tjian. 1988. Analysis of Spl in vivo reveals multiple transcriptional domains, including a novel glutamine-rich activation motif. Cell 55: 887-898.

Croston, G.E., L.A. Kerrigan, L.M. Lira, D.R. Marshak, and J.T. Kadonaga. 1991. Sequence-specific antirepression of histone Hl-mediated inhibition of basal RNA polymerase II transcription. Science 251: 643-649.

Dynlacht, B.D., T. Hoey, and R. Tjian. 1991. Isolation of coactivators associated with the TATA-binding protein that mediate transcriptional activation. Cell 55: 563-576.

Ellis, R.J. and S.M. Hemmingsen. 1989. Molecular chaperones: Proteins essential for the biogenesis of some macromolecular structures. Trends Biochem. Sci. 14: 339-342.

Flanagan, P.M., R.J. Kelleher III, M.H. Sayre, H. Tschochner, and R.D. Kornberg. 1991. A mediator required for activation of RNA polymerase II transcription in vitro. Nature 350: 436438.

Hahn, S., S. Buratowski, P.A. Sharp, and L. Guarente. 1989. Isolation of the gene encoding the yeast TATA binding protein TFIID: A gene identical to the SPT15 suppressor of Ty element insertion. Cell 58: 1173-1181.

Hoey, T., B.D. Dynlacht, M.G. Peterson, B.F. Pugh, and R. Tjian. 1990. Isolation and characterization of the Drosophila gene encoding the TATA box binding protein, TFIID. Cell 61: 1179-1186.

Hoffman, A., M. Horokoshi, C.K. Wang, S. Schroeder, P.A. Weil, and R.G. Roeder. 1990a. Cloning of the Schizosaccharomyces pombe TFIID gene reveals a strong conservation of functional domains present in Saccharomyces cerevisiae TFIID. Genes \& Dev. 4: 1141-1148.

Hoffman, A., E. Sinn, T. Yamamoto, J. Wang, A. Roy, M. Horikoshi, and R.G. Roeder. 1990b. Highly conserved core domain and unique $\mathrm{N}$ terminus with presumptive regulatory motifs in a human TATA factor (TFIID). Nature 346: 387390.

Horikoshi, M., C.K. Wang, H. Fujii, J.A. Cromlish, P.A. Weil, and R.G. Roeder. 1989a. Cloning and structure of a yeast gene encoding a general transcription initiation factor TFIID that binds to the TATA box. Nature 341: 299-301.

Horikoshi, M., C.K. Wang, H. Fujii, J.A. Cromlish, P.A. Weil and R.G. Roeder. 1989b. Purification of a yeast TATA boxbinding protein that exhibits human transcription factor IID activity. Proc. Natl. Acad. Sci. 86: 4843-4847.

Jones, K.A., J.T. Kadonaga, P.J. Rosenfeld, T.J. Kelly, and R. Tjian. 1987. A cellular DNA-binding protein that activates eukaryotic transcription and DNA replication. Cell 48: 79-
89.

Kao, C.C., P.M. Lieberman, M.C. Schmidt, Q. Zhou, R. Pei, and A.J. Berk. 1990. Cloning of a transcriptionally active human TATA binding factor. Science 248: 1646-1650.

Keegan, L., G. Gill, and M. Ptashne. 1986. Separation of DNA binding from the transcription-activating function of a eukaryotic transcriptional activator protein. Science 231: 699704.

Kelleher R.J. III, P.M. Flanagan, and R.D. Kornberg. 1990. A novel mediator between activator proteins and the RNA polymerase II transcription apparatus. Cell 61: 1209-1215.

Lillie, J.W. and M.R. Green. 1989. Transcription activation by the adenovirus Ela protein. Nature 338: $39-44$

Lin, Y.-S. and M.R. Green. 1991. Mechanism of action of an acidic transcriptional activator in vitro. Cell 64: 971-981.

Lin, Y.-S., M.F. Carey, M. Ptashne, and M.R. Green. 1988. GAL4 derivatives function alone and synergistically with mammalian activators in vitro. Cell 54: 659-664.

Maldonado, E., I. Ha, P. Cortese, L. Weis, and D. Reinberg. 1990. Factors involved in specific transcription by mammalian RNA polymerase II: Role of transcription factors IIA, IID, and IIB during formation of a transcription-competent complex. Mol. Cell. Biol. 10: 6335-6347.

Margottin, F., G. Dujardin, M. Gerard, J. Egly, J. Huet, and A. Sentenac. 1991. Participation of the TATA factor in transcription of the yeast U6 gene by RNA polymerase C. Science 251: 424-426.

Martin, K.J., J.W. Lillie, and M.R. Green. 1990. Evidence for interaction of different eukaryotic transcriptional activators with distinct cellular targets. Nature 346: 147-152.

Mermod, N., E.A. O'Neill, T.J. Kelly, and R. Tjian. 1989. The proline-rich transcriptional activator of CTF/NF-I is distinct from the replication and DNA binding domain. Cell 58: 741-753.

Mitchell, P.J. and R. Tjian. 1989. Transcriptional regulation in mammalian cells by sequence-specific DNA binding proteins. Science 245: 371-378.

Nakajima, N., M. Horikoshi, and R.G. Roeder. 1988. Factors involved in specific transcription by mammalian RNA polymerase II: Purification, genetic specificity, and TATA boxpromoter interactions of TFIID. Mol. Cell. Biol. 8: 40284040.

Peterson, M.G., N. Tanese, B.F. Pugh, and R. Tjian. 1990. Functional domains and upstream activation properties of a cloned human TATA binding protein. Science 248: 16251630.

Pugh, B.F. and R. Tiian. 1990. Mechanism of transcriptional activation by Spl: Evidence for coactivators. Cell 61: 11871197.

Reinberg, D. and R.G. Roeder. 1987. Factors involved in specific transcription by mammalian RNA polymerase II. J. Biol. Chem. 262: 3310-3321.

Reinberg, D., M. Horikoshi, and R.G. Roeder. 1987. Factors involved in specific transcription by RNA polymerase II. $I$. Biol. Chem. 262: 3322-3330.

Sadowski, I. and M. Ptashne. 1989. A vector for expressing GAL4 (1-147) fusions in mammalian cells. Nucleic Acids. Res. 17: 7539 .

Santoro, C., N. Mermod, P.C. Andrews, and R. Tjian. 1988. A family of human CCAAT-box-binding proteins active in transcription and DNA replication: Cloning and expression of multiple cDNAs. Nature 334: 218-224.

Sawadogo, M. and A. Sentenac. 1990. RNA polymerase B (II) and general transcription factors. Annu. Rev. Biochem. 59: 711754.

Schmidt, M.C., C.C. Kao, R. Pei, and A.J. Berk. 1989. Yeast 
Tanese et al.

TATA-box transcription factor gene. Proc. Natl. Acad. Sci. 86: $7785-7789$.

Simmen, K.A., J. Bernues, H.D. Parry, H.G. Stunnenberg, A. Berkenstam, B. Cavallini, J. Egly, and I.W. Mattaj. 1991. TFIID is required for in vitro transcription of the human U6 gene by RNA polymerase III. EMBO I. 10: 1853-1862.

Stringer, K.F., C.J. Ingles, and J. Greenblat. 1990. Direct and selective binding of an acidic transcriptional activation domain to the TATA-box factor TFIID. Nature 345: 783-786.

Van Dyke, M.W., R.G. Roeder, and M. Sawadago. 1988. Physical analysis of transcription preinitiation complex assembly on a class II gene promoter. Science 241: 1335-1338.

Workman, J.L., I.C.A. Taylor, and R.E. Kingston. 1991. Activation domains of stably bound GAL4 derivatives alleviate repression of promoters by nucleosomes. Cell 64: 533-544. 


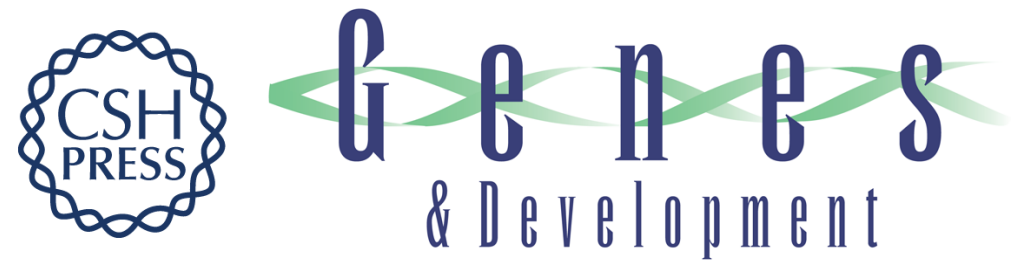

\section{Coactivators for a proline-rich activator purified from the multisubunit human TFIID complex.}

N Tanese, B F Pugh and R Tjian

Genes Dev. 1991, 5:

Access the most recent version at doi:10.1101/gad.5.12a.2212

References This article cites 44 articles, 16 of which can be accessed free at:

http://genesdev.cshlp.org/content/5/12a/2212.full.html\#ref-list-1

License

Email Alerting

Service

Receive free email alerts when new articles cite this article - sign up in the box at the top right corner of the article or click here.

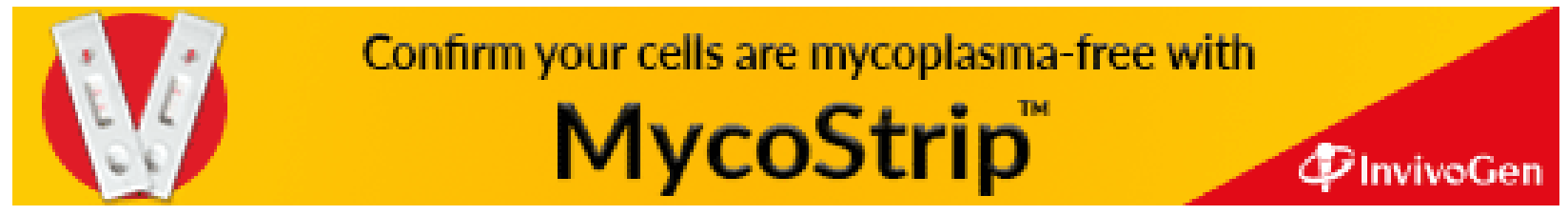

